\title{
A NeuN specific fluorescent probe revealing neuronal nuclei protein and nuclear acids association in living neurons under STED nanoscopy
}

\author{
Xiaohe Tian, ‡ab Tianyan Liu, ‡a Bin Fang, $\neq b$ Aidong Wang, c Mingzhu Zhang, b Sajid Hussain, a Lei \\ Luo, d Ruilong Zhang, b Qiong Zhang, b Jieying Wu,b Giuseppe Battaglia,e Zhongping Zhang*bf and \\ YupengTian*b
}

\begin{abstract}
Neuronal nuclei protein (NeuN) is a key RNA-associated protein to guide the transcription of RNA and regulate mRNA splicing in neurons. However, the lack of effective labelling and tracking method has hindered the elucidation of the biological mechanism of NeuN operation in living neurons to understand correlated central nervous system disorders. Here we report a molecular probe that can insert into a neighbouring hydrophobic-hydrophilic region in NeuN, which upon binding becomes capable of emitting light in the infrared. The NeuN specificity enables the probe imaging neuronal cells in primary brain regions including hippocampus, cerebellum, midbrain and cingulate gyrus. The probes optical properties are such to enable stimulated emission depletion (STED) imaging showing for the first time the 3D structure of RNA tangling into NeuN in a living neuron with tens of nanometer resolution.
\end{abstract}

\section{Introduction}

Neurons are the main component of the central nervous system (CNS) and they are well organized into complex brain structures including hippocampus, cerebellum and cingulate gyrus, responsible triggering the basic brain functions such as cognitive and emotional through electrical or chemical signals.[1] hence, effective neuron labelling remains a powerful tool to further understand the relevant functions of neurons in different brain domains. Up to date, wellestablished methods such as Immunofluorescence, [2] lipophilic tracers such as $\left(1,1^{\prime}\right.$-Dioctadecyl-3,3, 3', $3^{\prime}$ Tetramethylindocarbocyanine Perchlorate (Dil) [3]and viral vectors ${ }^{[4]}$ have been widely employed to label neurons for correlated investigation, however these approaches are often time consuming and pose risk to infect primary tissues with poor neuronal specificity. A recent effort was attempted using high-throughput screening to identify neuronal probes but the actual neuronal targeting mechanism remains unclear.[5]

We tackled this focusing our attention to a neuronal over-expressed protein, the neuronal nuclei protein (NeuN, also known as RBFOX3). [6] NeuN has been extensively used as mature neuron marker target (e.g. monoclonal antibodies A60) that specifically expressed only in neuronal nuclei and to some extent the cytoplasm of neuronal cells for most vertebrates, $[7]$ and to measure the neuron/glia ratio in brain regions. ${ }^{[8]}$ NeuN plays a prominent role in neural tissue development and regulation of adult brain function, while dysfunction or down regulation of NeuN have been associated with

a.School of Life Science, Anhui University, Hefei 230601, P. R. China.

b.Department of Chemistry, Anhui Province Key Laboratory of Chemistry for Inorganic/ Organic Hybrid Functionalized Materials, Anhui University, Hefei 230601, P. R. China.

c.School of Chemistry and Chemical Engineering, Huangshan University, Huangshan 245041, P. R. China

d.College of Pharmaceutical Sciences, Southwest University, Chongqing 400716, P. R. China

e.Department of Chemistry, University College London, London, WC1H OAJ, UK

f.CAS Center for Excellence in Nanoscience, Institute of Intelligent Machines, Chinese Academy of Sciences, Hefei 230000 , P. R. China.

¥These authors contributed equally.

Electronic Supplementary Information (ESI) available: [details of any supplementary information available should be included here]. See DOI: 10.1039/x0xx00000x several neurological disorders. ${ }^{[9]}$ Although NeuN is a nucleic acid binding protein,[10] its association with DNA or RNA has never been visualized by imaging. Therefore, staining live neurons in vivo or ex vivo by employing a small molecular probe with high specificity under fluorescent microscopy as well as its extended SR microscopy, could be a valuable approach for researchers.[11]

In this work, we reported a labelling method with a rationally designed molecular probe SL-Neu that specifically binds to NeuN in neurons. The probe fluorescence is only activated upon binding where the protein inhibits its configuration transfer and enhances infrared emission and two-photon absorption. The high selectivity of SL-Neu towards neuronal tissue and NeuN protein in contrast to other parenchymal cells was demonstrated. Due to its superb photo-stability against depletion laser power, we also showed for the first time the nuclear acid association (RNA and DNA) with NeuN protein in living neurons under 3-dimentional stimulated emission depletion nanoscopy (3D-STED). Such functional probe not only offers a powerful tool for specifically isolation neuronal cells, but also provides an insight on visualization and investigation of precise NeuN activities and correlated CNS disease for neurobiology.

\section{Results and discussion}

The chemical structures of the sulfosalt derivatives with $D-\pi-A$ ( $D=$ donor, $\mathrm{A}=$ acceptor) model including SL-Neu, SL-1, SL-2 and SL-3 were shown in Figure 1a. Their detailed synthesis, characterizations and crystal structural information were detailed in the ESI (Scheme S1, Figure S1-S15, Table S1-S3). Their photophysical properties, including nonlinear optical properties in the near-infrared region, were also systemically investigated (Figure S16-S22, TableS4-S5). It was found that the largest two-photon (2P) action cross-sections of probe SL-Neu in PBS buffer solution were located in the range of $840-940 \mathrm{~nm}$. The dual-hydroxyl groups in SL-Neu molecule have a tendency to form strong hydrogen-bind ( $\mathrm{H}$-bond) with NeuN amino residues. Furthermore, the introduction of sulfo-group favors water solubility (Figure S14) and biocompatibility of the compounds, which in turn allowed avoiding the use of biological unfriendly 
ARTICLE

solvents (e.g. DMSO) and at the same time offering the possibility to form $\mathrm{H}$-bind with NeuN protein, restricting molecular rotation.
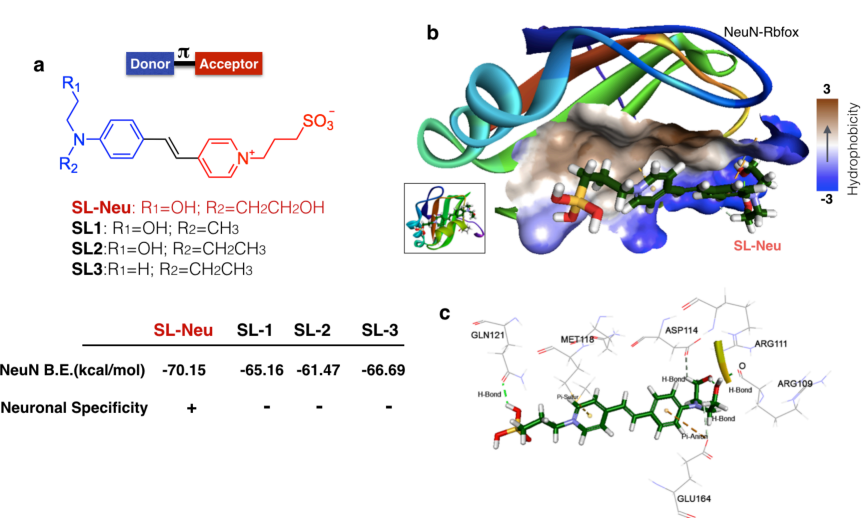

Fig. 1 (a) Chemical structure of sulfosalt derivatives including SLNeu, SL-1, SL-2 and SL-3; Insert table: modelled NeuN binding energy and live neuron specificity. (b) Molecular docking simulated hydrophobic and hydrophilic interaction of between SL-Neu and partial NeuN-Rbfox with indication of protein hydrophobicity; Insert figure: Molecular docking simulated diagram of SL-Neu and whole NeuN-Rbfox. (c) The detailed interaction of SL-Neu and amino acid residue in NeuN protein.

The specific recognition of SL-Neu and other compounds against NeuN-Rbfox protein (PDB: $2 \mathrm{~N} 82)^{13}$ was initially confirmed via molecular docking using the Discover Studio ligandfitVina software (version 2016, The Biovia Co.). ${ }^{14}$ The 2 N82 PDB structure possess a semi-open cavity consist by a $\alpha$-helix and a $\beta$-sheet, offering the possible binding site for SL-Neu molecule (Figure $1 \mathrm{~b}$ and Figure S23). In addition, the hydrophobic (Brown) hydrophilic (Blue) region in the protein active site (Figure $1 \mathrm{~b}$ ) interacts with corresponding hydrophobic/hydrophilic moieties of SL-Neu. Indeed, the detailed interactions including hydrogen binding and $\pi$-sulfur, $\pi$-anion (Figure 1c and Figure S24) was clearly presented (MET118, GLU164, GLN121, ASP114, ARG109). Notably, the relatively higher binding energy $(-70.159 \mathrm{Kcal} / \mathrm{mol})$ and neuronal specificity from SL-Neu molecule (Figure 1a insert table and Table S6) might be contributed by the dual-hydroxyl moieties, together with sulfonic acid group which led the formation of a 'triangle-like' $\mathrm{H}$-bind force within NeuN-Rbfox protein. Subsequently, the restricted rotation of such $D-\pi-A$ molecule may also led to enhanced intermolecular charge transfer (ICT), similarly as the influence of its solvent viscosity (Figure S25-S27). On the other hand, molecules SL1, SL2 and SL3, which lack the dual-hydroxyl moieties, have no neuronal selectivity at later ex vivo experiments.
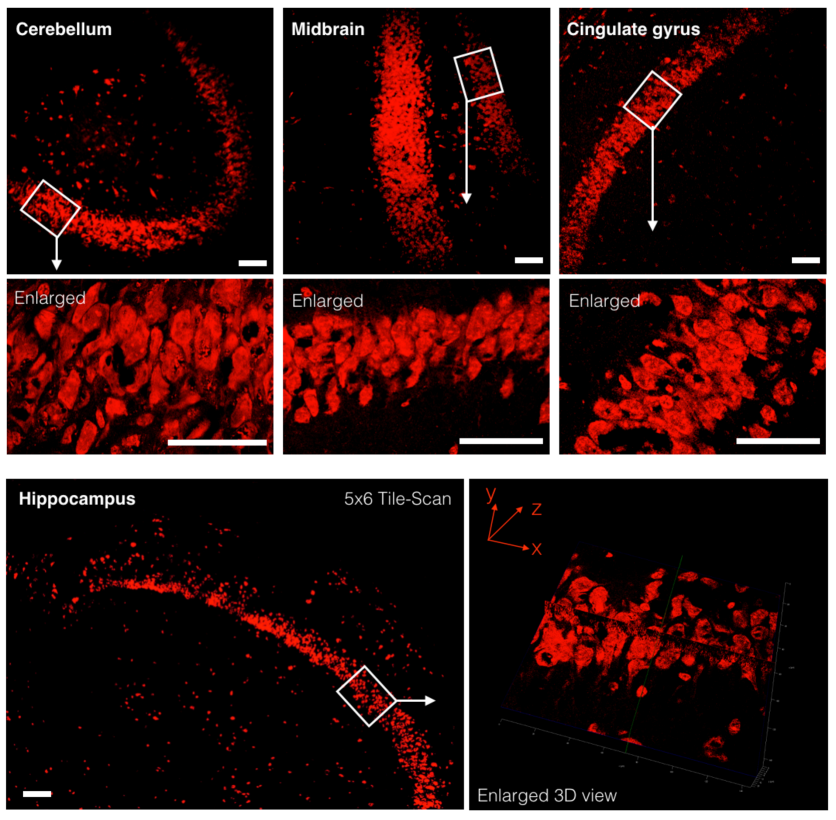

Fig. 2 Confocal images of cerebellum, midbrain, cingulate gyrus and hippocampus regions of fresh mouse brain sections incubated with SL-Neu compound $(10 \mu \mathrm{M})$ for $30 \mathrm{~min}$ with high magnification micrographs of the white rectangular regions, with 3-dimension image from selected region from hippocampus. Scale bar $=100 \mu \mathrm{m}$.

To elucidate the selectivity of these molecules for brain cells, fresh brain sections as well as liver, kidney and heart sections were prepared and incubated using SL-Neu, SL-1, SL-2 and SL-3 under 37 ${ }^{\circ} \mathrm{C}$ and $5 \% \mathrm{CO}_{2}$ for 1 hour. The primary tissue slices were then imaged under confocal laser scanning microscopy without further fixation. Notably, in liver, kidney and heart sections, the molecules produced negligible signal, while in SL-Neu treated brain sections some regions displayed strong emission (Figure S28). A similar brain uptake pattern was also observed under two-photon confocal microscopy (Figure S29). This reslut was also verified using prefixed tissues that were permeabilized with paraformaldehyde, suggesting that such specificity was present to both living and fixed samples. 
SL-Neu $\beta$-Tublin

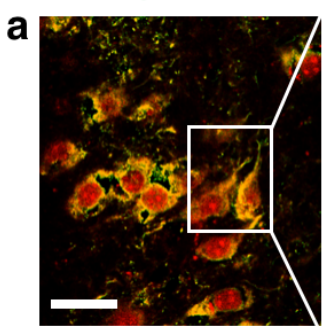

SL-Neu GFAP

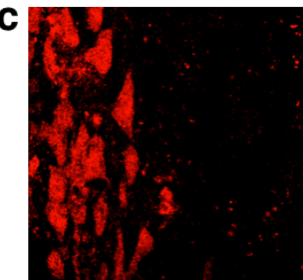

SL-Neu Capillary
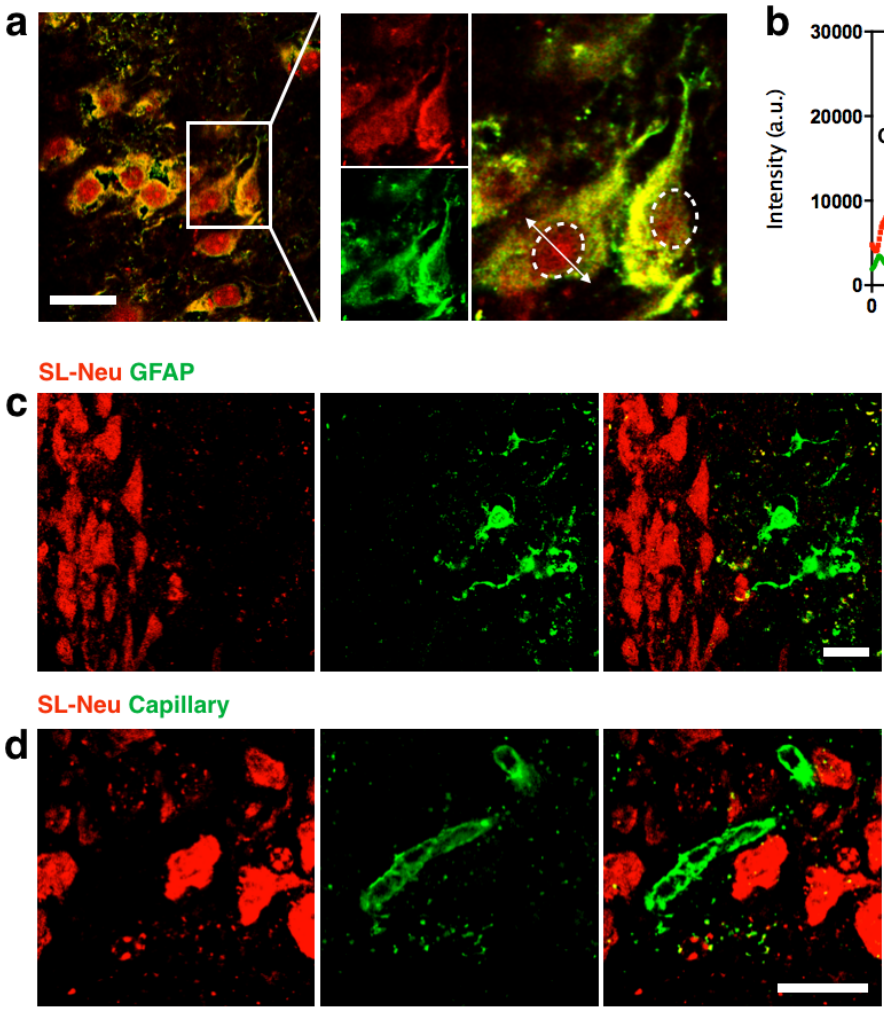

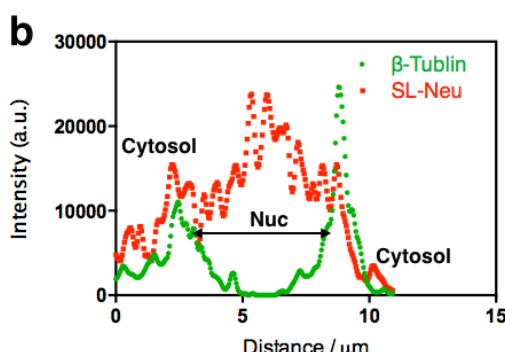

Distance / $\mu \mathrm{m}$

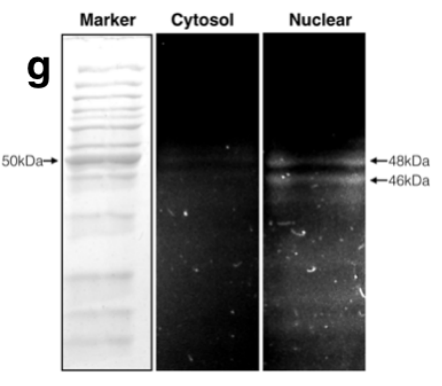

SL-Neu MAP2
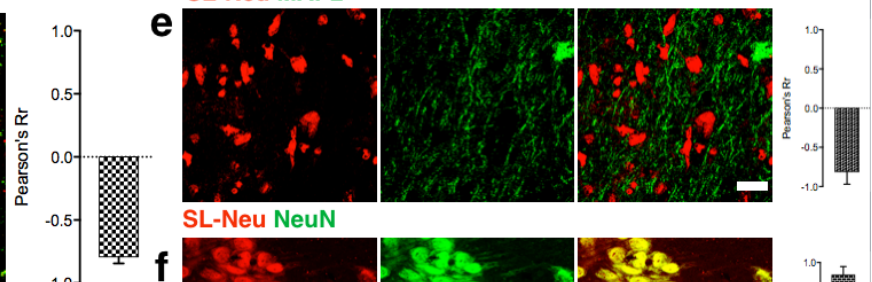

SL-Neu NeuN
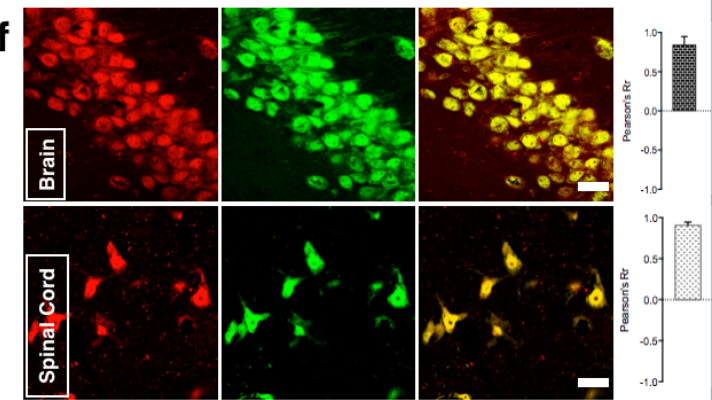

Fig. 3 Colocations studies of SL-Neu compound. (a) Left: Colocations micrographs between $\beta$-Tublin and SL-Neu compounds. Right: The magnified micrographs from selected region. (b) Single cell SL-Neu and $\beta$-Tublin intensity profile indicated the cellular distribution (c-e) The SL-Neu-stained regions were immunostained with GFAP for astrocytes, AlexaFluor-488 Lectin for Capillary, MAP-2 for neuronal fibrils, respectively. (f) SL-Neu-stained regions of mouse brain and spinal cord were immunostained with NeuN antibody. (g) SDS-PAGE analysis of protein from mouse nerve cell extracted from fresh brain hippocampus upon staining with Coomassie brilliant blue R-250 and SL-Neu. Coomassie brilliant blue R-250 staining (left). SL-Neu molecules staining under ultraviolet transillumination. Scale bar = $20 \mu \mathrm{m}$.

To further examine such specificity, brain sections with different neurons rich regions including cerebellum, midbrain, cingulate gyrus and hippocampus were evaluated. As Figure 2 and its enlarged micrographs showed, distinct signal was detected and these might highly correspondent to neuronal cells. Its neuronal specificity was confirmed when the brain cells were stained with the classic DNA dye DAPI (4', 6-diamidino-2-phenylindole), only cells within the hippocampus region displayed positive signal (Figure S30). In contrast, other cells (non-neuronal cells indicated by white arrows) showed negligible signals, suggesting the reduced uptake of SL-Neu molecules.

To verify the probe's intracellular distribution, $\beta$-Tublin antibody was used to label microtubules-rich neuron cells ${ }^{15}$ after incubating with SL-Neu molecule. As displayed in Figure $3 a$ in the magnified micrograph, $\beta$-Tublin demonstrated elongated neuronal fibril morphology and its signal were excluded from nuclear region. In contrast, SL-Neu displayed much less cytosolic distribution that partially overlapped with $\beta$-Tublin, while it showed intense nuclear internalization. The uptake pattern was further confirmed as intensity plot profile as indicated in Figure $3 \mathrm{~b}$. Such cytosolic and nuclear observation is not surprising, since NeuN protein could be both expressed only in neuronal nuclei and to some extent the cytoplasm. This further supports that SL-Neu compound was NeuN specific.

In order to confirm the selectivity of SL-Neu against neuronal NeuN protein, a series of immunofluorescence experiments were performed to label brain parenchymal cells and another neuronal marker. As one of the most representative cell population in the brain, astrocytes can be effectively stained using glial fibrillary acidic protein (GFAP) antibody. Figure 3c demonstrated a few astrocytes closely associated with SL-Neu-positive cells from hippocampus region but without overlapping, as indicated by co-localization coefficient analysis via Pearson's Rr (right bar-chart). Brian 


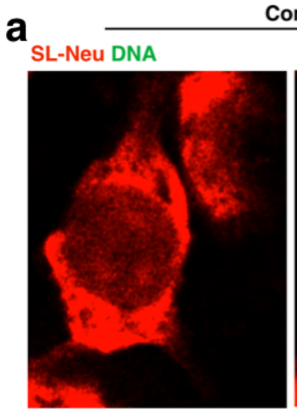

Confocal
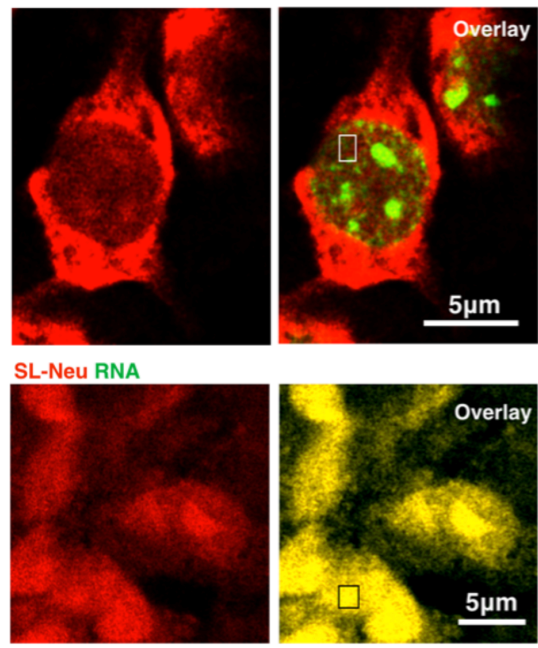

C

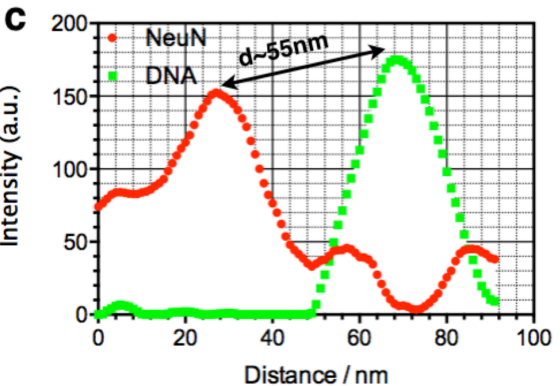

b
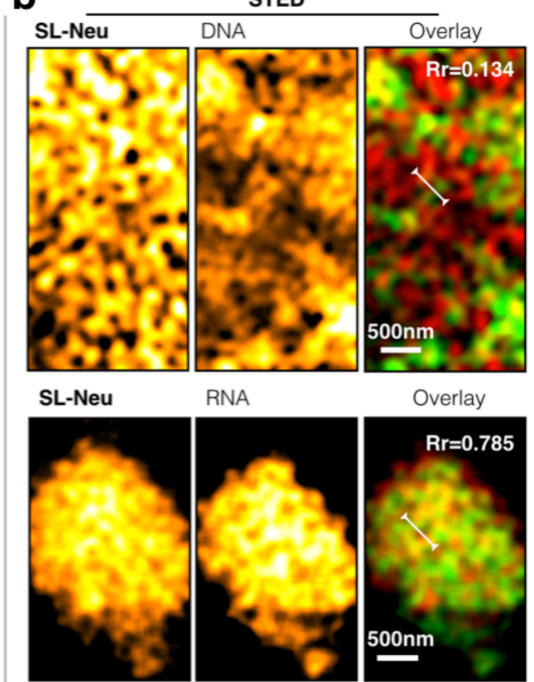

d 200

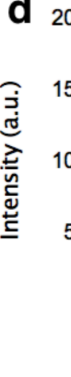

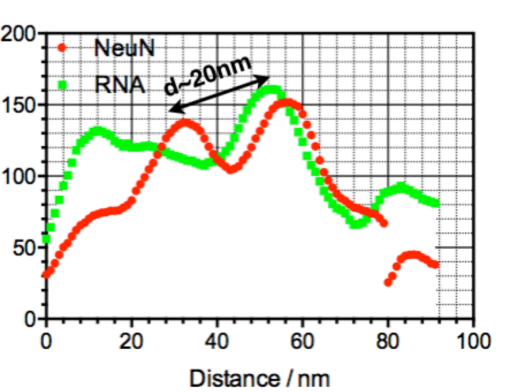

e
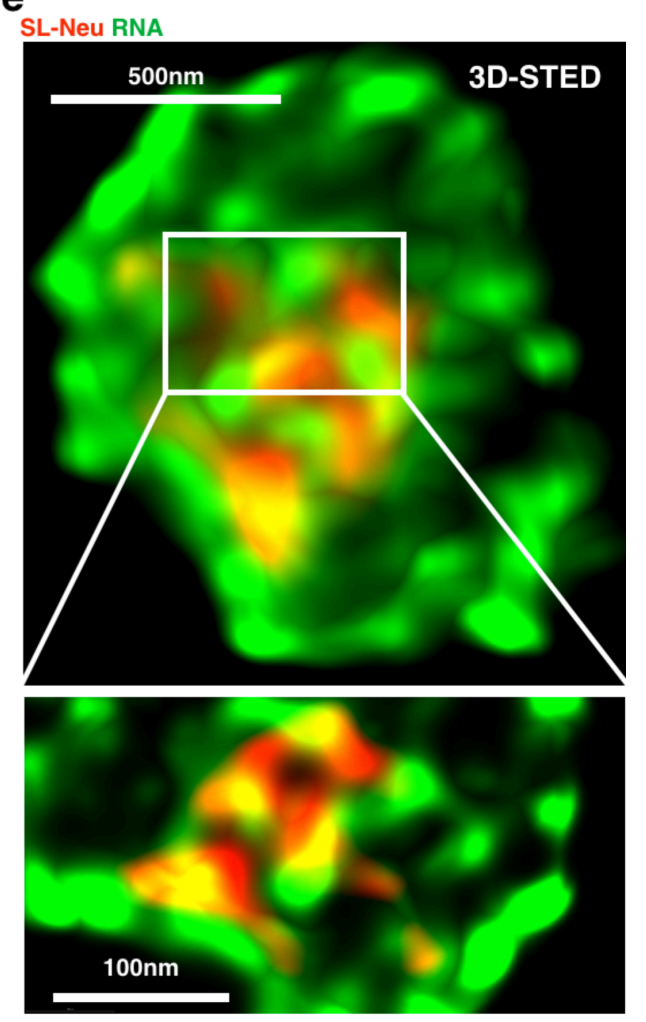

Fig. 4 (a) Confocal images of live neuron treated with SL-Neu and NucRed (DNA), Syto-9(RNA). (b) The corresponding STED images showing the combination of SL-Neu and DNA, RNA respectively under high resolution. (c) and (d) The fluorescent intensity profile of the lines in chromatin region. (e) and (f) 3-dimension STED micrographs indicating the association of SL-Neu and RNA.

endothelial cell is the main component of blood brain barrier that exist within brain parenchyma, responsible for substances exchange between central nervous system and the rest of body. As Figure 3d shown SL-Neu positive cells display no co-localization with blood vessels, again suggesting a very high specificity of SL-Neu molecule towards a specific cell type, which might correspond to neurons. Subsequently, MAP2 (microtubule-associated protein 2, a common marker of neuronal axons) was used to specifically label neuronal fibrils, and showed little overlap with SL-Neu signal.

Based on the aforementioned results such as strong intracellular fluorescence of SL-Neu in neurons rich regions, high intensity in nuclear region of $\beta$-tubulin rich neuronal cells, nonspecific interaction for non-neuronal cells and neuronal MAP2, costaining with NeuN antibody was eventually performed. Here brain and spinal cord of mouse were harvested, their slices were prepared and incubated with SL-Neu followed with standard immunofluorescence protocol. Figure $3 f$ clearly presents high degree of co-localization in both brain and spinal cord sections (Figure S31-S32). Generally, NeuN reactivity is mainly nuclear, whereas it may also be found in the cytoplasm of numerous neuronal cell types. Nonetheless, these immunofluorescence experiments strongly suggested SL-Neu molecules could effectively and specifically penetrate into live neuronal cells and internalize with neuronal nuclei protein, in a good agreement with our initial design purpose and computation modelling results.
To further confirm whether the staining of SL-Neu was indeed associated with the neuronal nuclei (NeuN) protein, nuclear and cytoplasmic proteins of mouse nerve cell were isolated from the hippocampus region, and protein electrophoresis was carried out. After electrophoresis, the gels were stained with SL-Neu $(10 \mu \mathrm{M})$ for $12 \mathrm{~h}$, and Coomassie brilliant blue was used as a reference. As shown in Figure $3 \mathrm{~g}$, SL-Neu molecules manifested the staining specificity of NeuN protein distributed in nucleus and cytoplasm by detecting two major bands (nuclear) and two weak bands (cytoplasm), highly correspondent to NeuN/Rbfox46- and 48-kDa subtypes. This result was in good agreement with imaging result, which showed SL-Neu signal and the NeuN protein signal with a considerably high overlay rate, again strongly suggesting SL-Neu was a NeuN specific fluorescent probe in either live or fixed neuronal cells.

The utilization of SL-Neu molecules under stimulated emission depletion (STED) nanoscopy to reveal the association between NeuN protein and nuclear acid was also performed. Although some literature data suggests that NeuN is a pre-mRNA alternative splicing regulator and binds to $\mathrm{RNA},{ }^{10}$ the correlation between NeuN and DNA or RNA in living neurons under super-resolution nanoscopy has never been demonstrated elsewhere, due to the lack of effective probes. Since STED technique require intense normal confocal laser along with a depletion laser, photo-stability is critical, 16 hence SL-Neu photo-resistance under STED condition was evaluated. Live brain section incubated with SL-Neu and exposed under STED laser over 400 -times continued scans. Intensity profile 
Journal Name

suggested such compound displayed super- stability under both confocal and STED condition (Figure S33).

Subsequently, in order to display the ultra-detailed correlations between SL-Neu and nuclear acid, we performed co-localization experiments using commercial dyes NucRed and Syto9 for DNA and RNA, respectively. Conventional live cell imaging was firstly obtained, suggesting the compatibility of SL-Neu molecules with those nuclear acids marker under confocal microscopy (Figure 4a and Figure S34). Due to the remarkable photo-stability and red emission of SL-Neu molecules, STED super resolution micrographs were captured. Figure $4 \mathrm{~b}$ indicated the nuclear NeuN protein was successfully marked with high signal-to-noise contrast. Neighboring chromatins structures were also revealed and the corresponding $x-y$ plot profile indicating a $\sim 55 \mathrm{~nm}$ distance between NeuN and DNA. In contrast, RNA showed much more overlapping signal with less than $\sim 20 \mathrm{~nm}$ distance was verified. Indeed, 3D super resolution single nucleolar imaging, with 85 Z-sections (Figure 4e), was successfully obtained and indicated the actual binding details of NeuN protein within the RNA entangled nucleoli. In contrast, DNA displayed much less interaction with SL-Neu signal (Figure S35). Although previous studies suggested NeuN protein could internalize with DNA in vitro, our observation using SL-Neu as a probe might strengthen that NeuN protein is a RNA associate protein in living neurons.

\section{Conclusions}

In summary, we have rationally designed a small fluorescent probe SL-Neu that can selectively target live neuron NeuN protein. SL-Neu live neuron labeling coupled with NeuN binding properties rendered further observation of NeuN and nuclear acid association using super-resolution technique under STED nanoscopy. This innovative compound serves as an imaging tool for living neurons as well as to shed light to correlated cellular mechanisms and ultimately NeuN associated CNS diseases.

\section{Conflicts of interest}

There are no conflicts to declare.

\section{Acknowledgements}

This work was supported by a grant for the National Natural Science Foundation of China (21602003, 51432001, 51672002, 51772002 and 21501001), Anhui University Doctor Startup Fund (J01001962). X.T thank Anhui Provincial Natural Science Foundation of China (1708085MC68) and Anhui Provincial Returnees Innovation and Entrepreneurship key support program. Giuseppe Battaglia thanks the Anhui Distinguished Foreign Expert program.

\section{Notes and references}

1. J. G. Nicholls, A. R. Martin, B. G. Wallace and P. A. Fuchs, From neuron to brain, Sinauer Associates Sunderland, MA, 2001.

2. A. Kádár, G. Wittmann, Z. Liposits and C. Fekete, Journal of neuroscience methods, 2009, 184, 115-118.

3. A. Schepartz, A. D. Thompson, M. H. Omar, F. Rivera-
ARTICLE

Molina, Z. Xi, A. J. Koleske and D. K. Toomre, Angewandte Chemie International Edition, 2017, 53, 10242-10246.

4. K. D. Foust, E. Nurre, C. L. Montgomery, A. Hernandez, C. M. Chan and B. K. Kaspar, Nature biotechnology, 2009, 27, 59.

5. J. C. Er, C. Leong, C. L. Teoh, Q. Yuan, P. Merchant, M. Dunn, D. Sulzer, D. Sames, A. Bhinge and D. Kim, Angewandte Chemie International Edition, 2015, 54, 2442-2446.

6. K. K. Kim, R. S. Adelstein and S. Kawamoto, Journal of Biological Chemistry, 2009, 284, 31052-31061.

7. A. Weyer and K. Schilling, Journal of neuroscience research, 2003, 73, 400-409.

8. S. Herculano-Houzel and R. Lent, Journal of Neuroscience, 2005, 25, 2518-2521.

9. W. Duan, Y.-P. Zhang, Z. Hou, C. Huang, H. Zhu, C.-Q. Zhang and Q. Yin, Molecular neurobiology, 2016, 53, 1637-1647.

10. M. A. Dent, E. Segura-Anaya, J. Alva-Medina and A. Aranda-Anzaldo, FEBS letters, 2010, 584, 2767-2771.

11. H. M. Kim and B. R. Cho, Chemical reviews, 2015, 115, 5014-5055.

12. G. Vicidomini, P. Bianchini and A. Diaspro, Nature methods, 2018.

13. Y. Chen, L. Zubovic, F. Yang, K. Godin, T. Pavelitz, J. Castellanos, P. Macchi and G. Varani, Nucleic acids research, 2016, 44, 4381-4395.

14. D. S. Biovia, Discovery Studio Modeling Envirnment, Release 2016. San Diego: Dassault Systemes, 2016.

15. L. C. Kapitein and C. C. Hoogenraad, Neuron, 2015, 87, 492-506.

16. L. Schermelleh, R. Heintzmann and $\mathrm{H}$. Leonhardt, The Journal of cell biology, 2010, 190, 165-175. 